\title{
BOUNDEDNESS OF SOME SUBLINEAR OPERATORS IN WEIGHTED HERZ-TYPE SPACES
}

\author{
SHANZHEN LU*, Kôzô YABUta ${ }^{\dagger}$ AND DaChun YanG ${ }^{\ddagger}$
}

\begin{abstract}
In this paper, the authors first establish the boundedness of sublinear operators on the weighted Herz space with general weights. At the extreme case, the authors show these operators are bounded from the weighted Herz space to the weighted weak Herz space. Moreover, the authors also discuss the boundedness of the local CalderonZygmund operator of the non-convolution type on the weighted Herz-type Hardy spaces and show that these operators map the weighted Herz-type Hardy space into the werghted weak Herz-type Hardy space at the extreme case.
\end{abstract}

\section{Introduction}

Let $B_{k}=B\left(0,2^{k}\right)=\left\{x \in \boldsymbol{R}^{n}:|x| \leq 2^{k}\right\}$ for any $k \in \boldsymbol{Z}$ and $\boldsymbol{R}_{k}=B_{k} \backslash B_{k-1}$. The following weighted Herz space is introduced by $\mathrm{Lu}$ and Yang in [11].

Definition 1. Let $\alpha \in \boldsymbol{R}, 0<p, q \leq \infty, \omega_{1}$ and $\omega_{2}$ be any non-negative weight functions.

(a) The homogeneous weighted Herz space $\dot{K}_{q}^{\alpha, p}\left(\omega_{1}, \omega_{2}\right)$ is defined by $\dot{K}_{q}^{\alpha, p}\left(\omega_{1}, \omega_{2}\right)=\left\{f: f\right.$ is a measurable function on $\boldsymbol{R}^{n}$ and $\left.\|f\|_{K_{q}^{\alpha, p}\left(\omega_{1}, \omega_{2}\right)}<\infty\right\}$, where

$$
\|f\|_{K_{q}^{\alpha, p}\left(\omega_{1}, \omega_{2}\right)}=\left\{\sum_{k=-\infty}^{\infty}\left[\omega_{1}\left(\boldsymbol{B}_{k}\right)\right]^{\alpha p / n}\left\|f \chi_{R_{k}}\right\|_{L_{\omega_{2}}^{q}\left(\boldsymbol{R}^{n}\right)}^{p}\right\}^{1 / p}
$$

with the usual modifications when $p=\infty$ and/or $q=\infty$.

1991 Mathematics Subject Classificatıon: Prımary 42B20, Secondary 42B30

Key words and phrases: Herz space, Hardy space, weak Herz space, weak Hardy space, Calderón-Zygmund operator, weight

* Shanzhen Lu is partially supported by the NNSF and the SEDF of China.

${ }^{\dagger}$ Kôzô Yabuta is supported by the Grant-1n-A1d for Basıc Scientific Research (10440046), Ministry of Education, Science and Culture, Japan.

${ }^{\ddagger}$ Dachun Yang is partially supported by the NNSF and the SEDF of China and the Grant-1n-A1d for Basic Scientific Research (10440046), Ministry of Education, Science and Culture, Japan.

Recelved September 3, 1999; revised October 29, 1999. 
(b) The non-homogeneous weighted Herz space $K_{q}^{\alpha, p}\left(\omega_{1}, \omega_{2}\right)$ is defined by $K_{q}^{\alpha, p}\left(\omega_{1}, \omega_{2}\right)=\left\{f: f\right.$ is a measurable function on $\boldsymbol{R}^{n}$ and $\left.\|f\|_{K_{q}^{\alpha . p}\left(\omega_{1}, \omega_{2}\right)}<\infty\right\}$, where

$$
\|f\|_{K_{q}^{\alpha, p}\left(\omega_{1}, \omega_{2}\right)}=\left\{\left\|f \chi_{B_{0}}\right\|_{L_{\omega_{2}}^{q}\left(\boldsymbol{R}^{n}\right)}^{p}+\sum_{k=1}^{\infty}\left[\omega_{1}\left(B_{k}\right)\right]^{\alpha p / n}\left\|f \chi_{R_{k}}\right\|_{L_{\omega_{2}}^{q}\left(\boldsymbol{R}^{n}\right)}^{p}\right\}^{1 / p}
$$

with the usual modifications when $p=\infty$ and/or $q=\infty$.

Here and in what follows, for any non-negative weight function $\omega$, any measurable function $f$ on $\boldsymbol{R}^{n}$ and any $q \in(0, \infty]$, we write

$$
\|f\|_{L_{\omega}^{q}\left(\boldsymbol{R}^{n}\right)}=\left\{\int_{\boldsymbol{R}^{n}}|f(x)|^{q} \omega(x) d x\right\}^{1 / q}
$$

and

$$
\|f\|_{W L_{\omega}^{q}\left(\boldsymbol{R}^{n}\right)}=\sup _{\lambda>0} \lambda\left[\omega\left(\left\{x \in \boldsymbol{R}^{n}:|f(x)|>\lambda\right\}\right)\right]^{1 / q}
$$

with the usual modification when $q=\infty$. If $\omega(x) \equiv 1$, we will denote $L_{\omega}^{q}\left(\boldsymbol{R}^{n}\right)$ and $W L_{\omega}^{q}\left(\boldsymbol{R}^{n}\right)$ simply by $L^{q}\left(\boldsymbol{R}^{n}\right)$ and $W L^{q}\left(\boldsymbol{R}^{n}\right)$.

Obviously, if $\alpha=0$, then $\dot{K}_{q}^{0, q}\left(\omega_{1}, \omega_{2}\right)=K_{q}^{0, q}\left(\omega_{1}, \omega_{2}\right)=L_{\omega_{2}}^{q}\left(\boldsymbol{R}^{n}\right)$ for any $q \in$ $(0, \infty]$. In what follows, if $\omega_{1}(x) \equiv \omega_{2}(x) \equiv 1$, we will denote $\dot{K}_{q}^{\alpha, p}\left(\omega_{1}, \omega_{2}\right)$ and $K_{q}^{\alpha, p}\left(\omega_{1}, \omega_{2}\right)$ simply by $\dot{K}_{q}^{\alpha, p}\left(\boldsymbol{R}^{n}\right)$ and $K_{q}^{\alpha, p}\left(\boldsymbol{R}^{n}\right)$.

Let $T$ be a sublinear operator satisfying that for any integrable function $f$ with a compact support and $x \notin \operatorname{supp} f$,

$$
|T f(x)| \leq c \int_{\boldsymbol{R}^{n}} \frac{|f(y)|}{|x-y|^{n}} d y,
$$

where $c$ is independent of $f$ and $x$. In [11], [10] and [8], such a sublinear operator is proved to be bounded on $\dot{K}_{q}^{\alpha, p}\left(\boldsymbol{R}^{n}\right)$ and $K_{q}^{\alpha, p}\left(\boldsymbol{R}^{n}\right)$ provided $T$ is bounded on $L^{q}\left(\boldsymbol{R}^{n}\right), 1<q<\infty, 0<p \leq \infty$ and $-n / q<\alpha<n(1-1 / q)$. Some weighted version of this is also considered by Lu and Yang in [11]. The first target of this paper will extend the result in [11]. In other words, we will much relax the restriction on the weights; see the following Theorem 1 .

Also, a sublinear operator satisfying (1) and being bounded on $L^{q}\left(\boldsymbol{R}^{n}\right)$ maybe is not bounded on $\dot{K}_{q}^{\alpha, p}\left(\boldsymbol{R}^{n}\right)$ or $K_{q}^{\alpha, p}\left(\boldsymbol{R}^{n}\right)$ for $0<p \leq \infty, 1<q<\infty$ and $\alpha=$ $-n / q$ or $\alpha=n(1-1 / q)$; see [11], [8] and [10]. However, in [6] and [7], $\mathrm{Hu}, \mathrm{Lu}$ and Yang introduced the weak Herz space and proved that such an operator is indeed bounded from $\dot{K}_{q}^{n(1-1 / q), p}\left(\boldsymbol{R}^{n}\right)$ to $W \dot{K}_{q}^{n(1-1 / q), p}\left(\boldsymbol{R}^{n}\right)$ or from $K_{q}^{n(1-1 / q), p}\left(\boldsymbol{R}^{n}\right)$ to $W K_{q}^{n(1-1 / q), p}\left(\boldsymbol{R}^{n}\right)$ if $0<p \leq 1$ and $1<q<\infty$. But, this is not true for $\alpha=$ $-n / q$ or $p>1$; see [7] for some counter-examples. The second purpose of this paper is to establish the weighted versions of these results in these extreme cases. First, we introduce the following weighted weak Herz space. 
Let $\omega_{2}$ be any non-negative weight function. For $k \in \boldsymbol{Z}, \sigma>0$ and any measurable function $f$ on $\boldsymbol{R}^{n}$, we define

$$
D_{k, \omega_{2}}(\sigma, f)=\omega_{2}\left(\left\{x \in R_{k}:|f(x)|>\sigma\right\}\right) ;
$$

for $k \in N$, let $\tilde{D}_{k \cdot \omega_{2}}(\sigma, f)=D_{k, \omega_{2}}(\sigma, f)$ and

$$
\tilde{D}_{0, \omega_{2}}(\sigma, f)=\omega_{2}\left(\left\{x \in B_{0}:|f(x)|>\sigma\right\}\right) .
$$

Definition 2. Let $\alpha \in \boldsymbol{R}, 0<q<\infty, 0<p \leq \infty$ and $\omega_{1}, \omega_{2}$ be any nonnegative weight functions.

(i) A measurable function $f$ on $\boldsymbol{R}^{n}$ is said to belong to the homogeneous weighted weak Herz space $W \dot{K}_{q}^{\alpha, p}\left(\omega_{1}, \omega_{2}\right)$ if

$$
\|f\|_{W K_{q}^{\alpha}{ }^{\alpha}\left(\omega_{1}, \omega_{2}\right)}=\sup _{\lambda>0} \lambda\left\{\sum_{k=-\infty}^{\infty}\left[\omega_{1}\left(B_{k}\right)\right]^{\alpha p / n}\left[D_{k, \omega_{2}}(\lambda, f)\right]^{p / q}\right\}^{1 / p}<\infty
$$

with the usual modification made when $p=\infty$.

(ii) A measurable function $f$ on $\boldsymbol{R}^{n}$ is said to belong to the non-homogeneous weighted weak Herz space $W K_{q}^{\alpha, p}\left(\omega_{1}, \omega_{2}\right)$ if

$$
\|f\|_{W K_{q}^{\alpha . p}\left(\omega_{1}, \omega_{2}\right)}=\sup _{\lambda>0} \lambda\left\{\sum_{k=0}^{\infty}\left[\omega_{1}\left(B_{k}\right)\right]^{\alpha p / n}\left[\tilde{D}_{k, \omega_{2}}(\lambda, f)\right]^{p / q}\right\}^{1 / p}<\infty
$$

with the usual modification made when $p=\infty$.

If $\omega_{1}(x) \equiv \omega_{2}(x) \equiv 1$, we will denote $W \dot{K}_{q}^{\alpha, p}\left(\omega_{1}, \omega_{2}\right)$ and $W K_{q}^{\alpha, p}\left(\omega_{1}, \omega_{2}\right)$ simply by $W \dot{K}_{q}^{\alpha, p}\left(\boldsymbol{R}^{n}\right)$ and $W K_{q}^{\alpha, p}\left(\boldsymbol{R}^{n}\right)$ which are introduced by $\mathrm{Hu}, \mathrm{Lu}$ and Yang in [6] and [7]. Also, $W \dot{K}_{q}^{0, q}\left(\omega_{1}, \omega_{2}\right)=W K_{q}^{0, q}\left(\omega_{1}, \omega_{2}\right)=W L_{\omega_{2}}^{q}\left(\boldsymbol{R}^{n}\right)$ for any $q \in(0, \infty)$.

The third purpose of this paper is to relax the restriction on the weight of the weighted Herz-type Hardy spaces studied in [12]. That is, we shall establish the atomic decomposition for the weighted Herz-type Hardy space with more general weights. Using this atomic decomposition, we shall establish the boundedness of local Caldrón-Zygmund operators of non-convolutional type from these weighted Herz-type Hardy sapces into weighted Herz spaces or into weighted weak Herz spaces at the extreme cases. Moreover, if we further suppose that these operators satisfy a vanishing moment condition, we then shall show that they are indeed bounded on the weighted Herz-type Hardy space or, in the extreme case, from the weighted Herz-type Hardy space into the weighted weak Herz-type Hardy space whose definition will be given later. Our results of this part extend the corresponding results in [7] to both non-convolutional types and weighted versions.

Finally, we recall the definition of the weight as follows. Let $1<p<\infty$. Following [5], a weight $\omega \geq 0$ is a Muckenhoupt $A_{p}\left(\boldsymbol{R}^{n}\right)$ weight if for any ball $B$

$$
\left(\frac{1}{|B|} \int_{B} \omega(x) d x\right)\left(\frac{1}{|B|} \int_{B}[\omega(x)]^{-1 /(p-1)} d x\right)^{p-1} \leq c
$$


with $c$ a constant independent of the ball $B$. The class $A_{1}\left(\boldsymbol{R}^{n}\right)$ is defined by letting $p \rightarrow 1$, namely,

$$
\frac{1}{|B|} \int_{B} \omega(x) d x \leq c \operatorname{essinf}_{x \in B} \omega(x)
$$

with $c$ independent of $B$. The smallest value of $c$ satisfying the above inequalities is called the $A_{p}\left(\boldsymbol{R}^{n}\right)$-constant of $\omega$. The following properties for $A_{p}\left(\boldsymbol{R}^{n}\right)$ weights will be repeatedly used in this paper; see [5], [15] for their proofs.

Lemma 1. Let $\omega \in A_{p}\left(\boldsymbol{R}^{n}\right)$ for some $p \in[1, \infty)$ and $B$ be any ball. Then (i) for any measurable function $f$ on $B$,

$$
|B|^{-1} \int_{B}|f(x)| d x \leq c[\omega(B)]^{-1 / p}\left(\int_{B}|f(x)|^{p} \omega(x) d x\right)^{1 / p}
$$

where $c$ is independent of $f$ and $B$;

(ii) if $E$ is a measurable subset of $B$, then

$$
\frac{\omega(B)}{\omega(E)} \leq c\left(\frac{|B|}{|E|}\right)^{p}
$$

where $c$ is independent of $B$ and $E$;

(iii) there exists a $\delta>0$ such that if $E$ is a measurable subset of $B$, then

$$
\frac{\omega(E)}{\omega(B)} \leq c\left(\frac{|E|}{|B|}\right)^{\delta}
$$

where $c$ is independent of $B$ and $E$.

Throughout this paper, $c$ always denotes a constant which is independent of the main parameters, but may vary from line to line.

We also remark that there is a similar result on the non-homogeneous Herztype space for any of our result on the homogeneous Herz-type space. For simplicity, we only state our results in the homogeneous Herz-type version.

Acknowledgement. The authors would like to express their deep thanks to the referee for his/her several valuable comments on this paper.

\section{Boundedness on weighted Herz spaces}

We begin with the boundedness on the weighted Herz space $\dot{K}_{q}^{\alpha, p}\left(\omega_{1}, \omega_{2}\right)$ for the sublinear operator satisfying certain "size" conditions.

THEOREM 1. Let $\omega_{1} \in A_{q_{\omega_{1}}}\left(\boldsymbol{R}^{n}\right), \omega_{2} \in A_{q_{\omega_{2}}}\left(\boldsymbol{R}^{n}\right), 0<p \leq \infty$ and $1<q<\infty$. If a sublinear operator $T$ is bounded on $L_{\omega_{2}}^{q}\left(\boldsymbol{R}^{n}\right)$ and satisfies (1), then $T$ is also bounded on $\dot{K}_{q}^{\alpha, p}\left(\omega_{1}, \omega_{2}\right)$ provided that $\omega_{1}$ and $\omega_{2}$ satisfy either of the following 
(i) $\omega_{1}=\omega_{2}, 1 \leq q_{\omega_{1}} \leq q$ and $-n q_{\omega_{1}} / q<\alpha q_{\omega_{1}}<n\left(1-q_{\omega_{1}} / q\right)$;

(ii) $1 \leq q_{\omega_{1}}<\infty, 1 \leq q_{\omega_{2}} \leq q$ and $0<\alpha q_{\omega_{1}}<n\left(1-q_{\omega_{2}} / q\right)$.

Proof. In what follows, let $\chi_{k}=\chi_{R_{k}}$ for any $k \in Z$. We write

$$
\begin{aligned}
\|T f\|_{K_{q}^{\alpha, p}\left(\omega_{1}, \omega_{2}\right)}= & \left\{\sum_{k=-\infty}^{\infty}\left[\omega_{1}\left(B_{k}\right)\right]^{\alpha p / n}\left\|(T f) \chi_{k}\right\|_{L_{\omega_{2}}^{q}\left(\boldsymbol{R}^{n}\right)}^{p}\right\}^{1 / p} \\
\leq & c\left\{\sum_{k=-\infty}^{\infty}\left[\omega_{1}\left(B_{k}\right)\right]^{\alpha p / n}\left(\sum_{l=-\infty}^{k-3}\left\|\chi_{k}\left(T f \chi_{l}\right)\right\|_{L_{\omega_{2}}^{q}\left(\boldsymbol{R}^{n}\right)}\right)^{p}\right\}^{1 / p} \\
& +c\left\{\sum_{k=-\infty}^{\infty} \cdots\left(\sum_{l=k-2}^{k+2} \cdots\right)^{p}\right\}^{1 / p}+\left\{\sum_{k=-\infty}^{\infty} \cdots\left(\sum_{l=k+3}^{\infty} \cdots\right)^{p}\right\}^{1 / p} \\
\equiv & E_{1}+E_{2}+E_{3} .
\end{aligned}
$$

By the $L_{\omega_{2}}^{q}\left(\boldsymbol{R}^{n}\right)$-boundedness of $T$, we are easy to obtain a desirable estimate for $E_{2}$.

For $E_{1}$, when $x \in R_{k}$ and $l \leq k-3$, by (1) and Hölder's inequality, we have

$$
\begin{aligned}
\left|T\left(f \chi_{l}\right)(x)\right| & \leq c \int_{R_{l}} \frac{|f(y)|}{|x-y|^{n}} d y \\
& \leq \frac{c}{2^{k n}} \int_{R_{l}}|f(y)| d y \\
& \leq \frac{c}{2^{k n}}\left\|f \chi_{l}\right\|_{L_{\omega_{2}}^{q}\left(\boldsymbol{R}^{n}\right)}\left(\int_{B_{l}}\left[\omega_{2}(x)\right]^{-1 /(q-1)} d x\right)^{1 / q^{\prime}} \\
& \leq c 2^{(l-k) n}\left\|f \chi_{l}\right\|_{L_{\omega_{2}}^{q}\left(\boldsymbol{R}^{n}\right)} \frac{1}{\left[\omega_{2}\left(B_{l}\right)\right]^{1 / q}},
\end{aligned}
$$

since $\omega_{2} \in A_{q}\left(\boldsymbol{R}^{n}\right)$. Thus, by $\alpha q_{\omega_{1}}<n\left(1-q_{\omega_{2}} / q\right)$, we have

$$
\begin{aligned}
E_{1} & \leq c\left\{\sum_{k=-\infty}^{\infty}\left(\sum_{l=-\infty}^{k-3}\left[\omega_{1}\left(B_{l}\right)\right]^{\alpha / n}\left\|f \chi_{l}\right\|_{L_{\omega_{2}}^{q}\left(\boldsymbol{R}^{n}\right)} 2^{(l-k) n} \times\left[\frac{\omega_{1}\left(B_{k}\right)}{\omega_{1}\left(B_{l}\right)}\right]^{\alpha / n}\left[\frac{\omega_{2}\left(B_{k}\right)}{\omega_{2}\left(B_{l}\right)}\right]^{1 / q}\right)^{p}\right\}^{1 / p} \\
& \leq c\left\{\sum_{k=-\infty}^{\infty}\left(\sum_{l=-\infty}^{k-3}\left[\omega_{1}\left(B_{l}\right)\right]^{\alpha / n}\left\|f \chi_{l}\right\|_{L_{\omega_{2}}^{q}\left(\boldsymbol{R}^{n}\right)} \times 2^{(k-l)\left(\alpha q_{\omega_{1}}+n q_{\omega_{2}} / q-n\right)}\right)^{p}\right\}^{1 / p}
\end{aligned}
$$




$$
\begin{aligned}
& \int\left\{\sum _ { k = - \infty } ^ { \infty } \left(\sum_{l=-\infty}^{k-3}\left[\omega_{1}\left(B_{l}\right)\right]^{\alpha p / n}\left\|f \chi_{l}\right\|_{L_{\omega_{2}}^{q}\left(\boldsymbol{R}^{n}\right)}^{p}\right.\right. \\
& \left.\left.\times 2^{(k-l)\left(\alpha q_{\omega_{1}}+n q_{\omega_{2}} / q-n\right) p}\right)\right\}^{1 / p}, \quad \text { if } 0<p \leq 1
\end{aligned}
$$

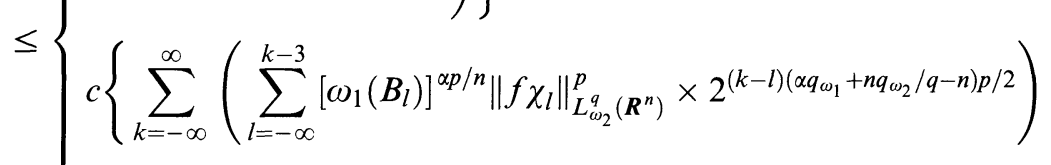

$$
\begin{aligned}
& \left.\times\left(\sum_{l=-\infty}^{k-3} 2^{(k-l)\left(\alpha q_{\omega_{1}}+n q_{\omega_{2}} / q-n\right) p^{\prime} / 2}\right)^{p / p^{\prime}}\right\}^{1 / p}, \quad \text { if } 1<p<\infty \\
& \int\left\{\sum_{l=-\infty}^{\infty}\left[\omega_{1}\left(B_{l}\right)\right]^{\alpha p / n}\left\|f \chi_{l}\right\|_{L_{\omega_{2}}^{q}\left(\boldsymbol{R}^{n}\right)}^{p}\right. \\
& \leq\left\{\begin{aligned}
&\left.\times\left(\sum_{k=l+3}^{\infty} 2^{(k-l)\left(\alpha q_{\omega_{1}}+n q_{\omega_{2}} / q-n\right) p}\right)\right\}^{1 / p}, \quad \text { if } 0<p \leq 1 \\
& c\left\{\sum_{l=-\infty}^{\infty}\left[\omega_{1}\left(B_{l}\right)\right]^{\alpha p / n}\left\|f \chi_{l}\right\|_{L_{\omega_{2}}^{q}\left(\boldsymbol{R}^{n}\right)}^{p}\right.
\end{aligned}\right. \\
& \left.\times\left(\sum_{k=l+3}^{\infty} 2^{(k-l)\left(\alpha q_{\omega_{1}}+n q_{\omega_{2}} / q-n\right) p / 2}\right)\right\}^{1 / p}, \quad \text { if } 1<p<\infty \\
& \leq c\left\{\sum_{l=-\infty}^{\infty}\left[\omega_{1}\left(B_{l}\right)\right]^{\alpha p / n}\left\|f \chi_{l}\right\|_{L_{\omega_{2}}^{q}\left(\boldsymbol{R}^{n}\right)}^{p}\right\}^{1 / p} \\
& =c\|f\|_{K_{q}^{\alpha, p}\left(\omega_{1}, \omega_{2}\right)},
\end{aligned}
$$

where, and in what follows, $1 / p+1 / p^{\prime}=1$ and we used the fact that $\alpha+n / q_{1}$ $>0$ when $\omega_{1}=\omega_{2}$ and $\alpha>0$ when $\omega_{1} \neq \omega_{2}$. So far, we have obtained a desirable estimate for $E_{1}$.

For the estimate of $E_{3}$, when $x \in R_{k}$ and $l \geq k+3$, by (1), we have

$$
\begin{aligned}
\left|T\left(f \chi_{l}\right)(x)\right| & \leq c \int_{R_{l}} \frac{|f(y)|}{|x-y|^{n}} d y \\
& \leq \frac{c}{2^{\ln }} \int_{R_{l}}|f(y)| d y \\
& \leq \frac{c}{2^{l n}}\left\|f \chi_{l}\right\|_{L_{\omega_{2}}^{q}\left(\boldsymbol{R}^{n}\right)}\left(\int_{B_{l}}\left[\omega_{2}(x)\right]^{-1 /(q-1)} d x\right)^{1 / q^{\prime}} \\
& \leq c \frac{\left\|f \chi_{l}\right\|_{L_{\omega_{2}}^{q}\left(\boldsymbol{R}^{n}\right)}}{\left[\omega_{2}\left(B_{l}\right)\right]^{1 / q}}
\end{aligned}
$$


since $\omega_{2} \in A_{q}\left(\boldsymbol{R}^{n}\right)$. Thus, we have

$$
\begin{aligned}
& E_{3} \leq c\left\{\sum_{k=-\infty}^{\infty}\left(\sum_{l=k+3}^{\infty}\left[\omega_{1}\left(B_{l}\right)\right]^{\alpha / n}\left\|f \chi_{l}\right\|_{L_{\omega_{2}}^{q}\left(\boldsymbol{R}^{n}\right)}\left[\frac{\omega_{1}\left(B_{k}\right)}{\omega_{1}\left(B_{l}\right)}\right]^{\alpha / n}\left[\frac{\omega_{2}\left(B_{k}\right)}{\omega_{2}\left(B_{l}\right)}\right]^{1 / q}\right)^{p}\right\}^{1 / p} \\
& \leq c\left\{\sum_{k=-\infty}^{\infty}\left(\sum_{l=k+3}^{\infty}\left[\omega_{1}\left(B_{l}\right)\right]^{\alpha / n}\left\|f \chi_{l}\right\|_{L_{\omega_{2}}^{q}\left(\boldsymbol{R}^{n}\right)} 2^{(k-l) n\left(\delta_{1} \alpha / n+\delta_{2} / q\right)}\right)^{p}\right\}^{1 / p} \\
& \int c\left\{\sum _ { k = - \infty } ^ { \infty } \left(\sum_{l=k+3}^{\infty}\left[\omega_{1}\left(B_{l}\right)\right]^{\alpha p / n}\left\|f \chi_{l}\right\|_{L_{\omega_{2}}^{q}\left(\boldsymbol{R}^{n}\right)}^{p}\right.\right. \\
& \leq\left\{\begin{array}{c}
\left.\left.\times 2^{(k-l)\left(\delta_{1} \alpha+\delta_{2} n / q\right) p}\right)\right\}^{1 / p}, \quad \text { if } 0<p \leq 1 \\
c\left\{\sum _ { k = - \infty } ^ { \infty } \left(\sum_{l=k+3}^{\infty}\left[\omega_{1}\left(B_{l}\right)\right]^{\alpha p / n}\left\|f \chi_{l}\right\|_{L_{\omega_{2}}^{q}\left(\boldsymbol{R}^{n}\right)}^{p}\right.\right.
\end{array}\right. \\
& \left.\times 2^{(k-l)\left(\delta_{1} \alpha+\delta_{2} n / q\right) p / 2}\left(\sum_{l=k+3}^{\infty} 2^{(k-l)\left(\delta_{1} \alpha+\delta_{2} n / q\right) p^{\prime} / 2}\right)^{p / p^{\prime}}\right\}^{1 / p}, \text { if } 1<p<\infty \\
& \int c\left\{\sum_{l=-\infty}^{\infty}\left[\omega_{1}\left(B_{l}\right)\right]^{\alpha p / n}\left\|f \chi_{l}\right\|_{L_{\omega_{2}}^{q}\left(\boldsymbol{R}^{n}\right)}^{p}\right. \\
& \leq\left\{\begin{array}{c}
\left.\times\left(\sum_{k=-\infty}^{l-3} 2^{(k-l)\left(\delta_{1} \alpha+\delta_{2} n / q\right) p}\right)\right\}^{1 / p}, \text { if } 0<p \leq 1 \\
\left\{\sum^{\infty}\left[\omega_{1}\left(B_{l}\right)\right]^{\alpha p / n}\left\|f \chi_{l}\right\|_{L^{p}}{ }^{p}\right.
\end{array}\right. \\
& c\left\{\sum_{l=-\infty}^{\infty}\left[\omega_{1}\left(B_{l}\right)\right]^{\alpha p / n}\left\|f \chi_{l}\right\|_{L_{\omega_{2}}^{q}\left(\boldsymbol{R}^{n}\right)}^{p}\right. \\
& \left.\times\left(\sum_{k=-\infty}^{l-3} 2^{(k-l)\left(\delta_{1} \alpha+\delta_{2} n / q\right) p / 2}\right)\right\}^{1 / p}, \quad \text { if } 1<p<\infty \\
& \leq c\left\{\sum_{l=-\infty}^{\infty}\left[\omega_{1}\left(B_{l}\right)\right]^{\alpha p / n}\left\|f \chi_{l}\right\|_{L_{\omega_{2}}^{q}\left(\boldsymbol{R}^{n}\right)}^{p}\right\}^{1 / p} \\
& =c\|f\|_{K_{q}^{\alpha p}\left(\omega_{1}, \omega_{2}\right)},
\end{aligned}
$$

where $\delta_{1}>0, \delta_{2}>0$ depend only on $n$ and the $A_{q_{\omega_{1}}}\left(\boldsymbol{R}^{n}\right)$-constant and the $A_{q_{\omega_{2}}}\left(\boldsymbol{R}^{n}\right)$-constant, and $\delta_{1}=\delta_{2}$ when $\omega_{1}=\omega_{2}$.

We leave the case $p=\infty$ to the reader.

This finishes the proof of Theorem 1.

We remark that the condition (1) can be replaced by more general conditions; see $[10]$. 
On the end cases of Theorem 1, we have the following conclusion, which can be regarded as a weighted version of Theorem 1 in [7].

THEOREM 2. Let $\omega_{1}, \omega_{2} \in A_{1}\left(\boldsymbol{R}^{n}\right), 0<p \leq 1,1 \leq q<\infty$ and $\alpha=n(1-1 / q)$. If a sublinear operator $T$ is bounded from $L_{\omega_{2}}^{q}\left(\boldsymbol{R}^{n}\right)$ into $W L_{\omega_{2}}^{q}\left(\boldsymbol{R}^{n}\right)$ and satisfies (1), then $T$ is also bounded from $\dot{K}_{q}^{\alpha, p}\left(\omega_{1}, \omega_{2}\right)$ into $W \dot{K}_{q}^{\alpha, p}\left(\omega_{1}, \omega_{2}\right)$.

Proof. Let $f \in \dot{K}_{q}^{\alpha, p}\left(\omega_{1}, \omega_{2}\right)$ and for any $k \in \boldsymbol{Z}$, we write

$$
\begin{aligned}
f(x) & =f(x) \chi_{\left\{|x| \leq 2^{k-3}\right\}}(x)+f(x) \chi_{\left\{2^{k-3}<|x| \leq 2^{k+2}\right\}}(x)+f(x) \chi_{\left\{|x|>2^{k+2}\right\}}(x) \\
& \equiv f_{1}^{k}(x)+f_{2}^{k}(x)+f_{3}^{k}(x) .
\end{aligned}
$$

Then $|T f(x)| \leq\left|T f_{1}^{k}(x)\right|+\left|T f_{2}^{k}(x)\right|+\left|T f_{3}^{k}(x)\right|$, and

$$
\begin{aligned}
\|T f\|_{W K_{q}^{\alpha, p}\left(\omega_{1}, \omega_{2}\right)}= & \sup _{\lambda>0} \lambda\left\{\sum_{k=-\infty}^{\infty}\left[\omega_{1}\left(B_{k}\right)\right]^{\alpha p / n}\left[D_{k, \omega_{2}}(\lambda, T f)\right]^{p / q}\right\}^{1 / p} \\
= & c \sup _{\lambda>0} \lambda\left\{\sum_{k=-\infty}^{\infty}\left[\omega_{1}\left(B_{k}\right)\right]^{\alpha p / n}\left[D_{k, \omega_{2}}\left(\lambda / 3,\left|T f_{1}^{k}\right|\right)\right]^{p / q}\right\}^{1 / p} \\
& +c \sup _{\lambda>0} \lambda\left\{\sum_{k=-\infty}^{\infty}\left[\omega_{1}\left(B_{k}\right)\right]^{\alpha p / n}\left[D_{k, \omega_{2}}\left(\lambda / 3,\left|T f_{2}^{k}\right|\right)\right]^{p / q}\right\}^{1 / p} \\
& +c \sup _{\lambda>0} \lambda\left\{\sum_{k=-\infty}^{\infty}\left[\omega_{1}\left(B_{k}\right)\right]^{\alpha p / n}\left[D_{k, \omega_{2}}\left(\lambda / 3,\left|T f_{3}^{k}\right|\right)\right]^{p / q}\right\}^{1 / p} \\
\equiv & F_{1}+F_{2}+F_{3} .
\end{aligned}
$$

By the fact that $T$ is bounded from $L_{\omega_{2}}^{q}\left(\boldsymbol{R}^{n}\right)$ into $W L_{\omega_{2}}^{q}\left(\boldsymbol{R}^{n}\right)$, we easily obtain a desirable estimate for $F_{2}$.

To estimate $F_{1}$, for $x \in R_{k}$, by (1) and Minkowski's inequality, we have

$$
\begin{aligned}
\left|T f_{1}^{k}(x)\right| & \leq c \int_{\boldsymbol{R}^{n}} \frac{\left|f_{1}^{k}(y)\right|}{|x-y|^{n}} d y \\
& \leq\left\{\begin{array}{l}
\frac{c}{\omega_{2}\left(B_{k}\right)}\left\|f_{1}^{k}\right\|_{L_{\omega_{2}}^{1}\left(\boldsymbol{R}^{n}\right)}, \quad q=1 \\
c 2^{-k n} \sum_{j=-\infty}^{k-3}\left\|f \chi_{j}\right\|_{L_{\omega_{2}}^{q}\left(\boldsymbol{R}^{n}\right)}\left\{\int_{B_{j}}\left[\omega_{2}(x)\right]^{-1 /(q-1)} d x\right\}^{1 / q^{\prime}}, \quad q>1
\end{array}\right. \\
& \leq\left\{\begin{array}{l}
\frac{c}{\omega_{2}\left(B_{k}\right)}\left\|f_{1}^{k}\right\|_{L_{\omega_{2}}^{1}\left(\boldsymbol{R}^{n}\right)}, \quad q=1 \\
\frac{c}{2^{k n}} \sum_{j=-\infty}^{k-3} \frac{2^{j n}}{\left[\omega_{2}\left(B_{j}\right)\right]^{1 / q}}\left\|f \chi_{j}\right\|_{L_{\omega_{2}}^{q}\left(\boldsymbol{R}^{n}\right)}, \quad q>1
\end{array}\right.
\end{aligned}
$$




$$
\begin{aligned}
& \leq \frac{c_{1}}{\left[\omega_{1}\left(B_{k}\right)\right]^{\alpha / n}\left[\omega_{2}\left(B_{k}\right)\right]^{1 / q}} \sum_{J=-\infty}^{k-3}\left[\omega_{1}\left(B_{j}\right)\right]^{\alpha / n}\left\|f \chi_{J}\right\|_{L_{\omega_{2}}^{q}\left(\boldsymbol{R}^{n}\right)} \\
& \leq \frac{c_{1}}{\left[\omega_{1}\left(B_{k}\right)\right]^{\alpha / n}\left[\omega_{2}\left(B_{k}\right)\right]^{1 / q}}\|f\|_{K_{q}^{\alpha, p}\left(\omega_{1}, \omega_{2}\right)}
\end{aligned}
$$

since $\omega_{2} \in A_{q}\left(\mathbb{R}^{n}\right), p \leq 1$ and $\alpha=n(1-1 / q)$. Now, for any given $\lambda>0$, let $k_{\lambda}$ be the greatest integer satisfying

$$
\lambda / 3<\frac{c_{1}}{\left[\omega_{1}\left(B_{k}\right)\right]^{\alpha / n}\left[\omega_{2}\left(B_{k_{\lambda}}\right)\right]^{1 / q}}\|f\|_{K_{q}^{\alpha, p}\left(\omega_{1}, \omega_{2}\right)} .
$$

Then,

$$
\begin{aligned}
F_{1} & \leq c \sup _{\lambda>0} \lambda\left\{\sum_{k=-\infty}^{k_{\lambda}}\left[\omega_{1}\left(B_{k}\right)\right]^{\alpha p / n}\left[\omega_{2}\left(B_{k}\right)\right]^{p / q}\right\}^{1 / p} \\
& \leq c\|f\|_{K_{q}^{\alpha, p}\left(\omega_{1}, \omega_{2}\right)} \sup _{\lambda>0}\left\{\sum_{k=-\infty}^{k_{\lambda}}\left[\frac{\omega_{1}\left(B_{k}\right)}{\omega_{1}\left(B_{k_{\lambda}}\right)}\right]^{\alpha p / n}\left[\frac{\omega_{2}\left(B_{k}\right)}{\omega_{2}\left(B_{k_{\lambda}}\right)}\right]^{p / q}\right\}^{1 / p} \\
& \leq c\|f\|_{K_{q}^{\alpha, p}\left(\omega_{1}, \omega_{2}\right)} \sup _{\lambda>0}\left\{\sum_{k=-\infty}^{k_{,}} 2^{\left(k-k_{\lambda}\right)\left(\delta_{1} \alpha+\delta_{2} n / q\right) p}\right\}^{1 / p} \\
& \leq c\|f\|_{K_{q}^{\alpha, p}\left(\omega_{1}, \omega_{2}\right)},
\end{aligned}
$$

where $\delta_{1}>0$ and $\delta_{2}>0$ depend on $n$ and the $A_{1}\left(\boldsymbol{R}^{n}\right)$-constants of $\omega_{1}$ and $\omega_{2}$; see Lemma 1. This is a desirable estimate for $F_{1}$.

We now estimate $F_{3}$. For $x \in R_{k}$, by (1) we have

$$
\begin{aligned}
\left|T f_{3}^{k}(x)\right| & \leq c \int_{\boldsymbol{R}^{n}} \frac{\left|f_{3}^{k}(y)\right|}{|x-y|^{n}} d y \\
& \leq c \sum_{J=k+3}^{\infty} 2^{-\jmath n}\left\|f \chi_{j}\right\|_{L^{1}\left(\boldsymbol{R}^{n}\right)} \\
& \leq\left\{\begin{array}{l}
c \sum_{J=k+3}^{\infty} \frac{1}{\omega_{2}\left(B_{j}\right)}\left\|f \chi_{j}\right\|_{L_{\omega_{2}}^{1}\left(\boldsymbol{R}^{n}\right)}, \quad q=1 \\
c \sum_{J=k+3}^{\infty} 2^{-j n}\left\|f \chi_{j}\right\|_{L_{\omega_{2}}^{q}\left(\boldsymbol{R}^{n}\right)}\left(\int_{B_{J}}\left[\omega_{2}(x)\right]^{-1 /(q-1)} d x\right)^{1 / q^{\prime}}, \quad q>1
\end{array}\right. \\
& \leq c \sum_{j=k+3}^{\infty} \frac{1}{\left[\omega_{2}\left(B_{j}\right)\right]^{1 / q}}\left\|f \chi_{j}\right\|_{L_{\omega_{2}}^{q}\left(\boldsymbol{R}^{n}\right)}
\end{aligned}
$$




$$
\begin{aligned}
& \leq \frac{c}{\left[\omega_{1}\left(B_{k}\right)\right]^{\alpha / n}\left[\omega_{2}\left(B_{k}\right)\right]^{1 / q}} \sum_{J=k+3}^{\infty}\left[\omega_{1}\left(B_{j}\right)\right]^{\alpha / n}\left\|f \chi_{j}\right\|_{L_{\omega_{2}}^{q}\left(\boldsymbol{R}^{n}\right)} \\
& \leq \frac{c_{2}}{\left[\omega_{1}\left(B_{k}\right)\right]^{\alpha / n}\left[\omega_{2}\left(B_{k}\right)\right]^{1 / q}}\|f\|_{K_{q}^{\alpha, p}\left(\omega_{1}, \omega_{2}\right)},
\end{aligned}
$$

since $\omega_{2} \in A_{q}\left(\boldsymbol{R}^{n}\right), p \leq 1$ and $\alpha \geq 0$.

Now, similar to the estimate for $F_{1}$, we can show that

$$
F_{3} \leq c\|f\|_{K_{q}^{\alpha, p}\left(\omega_{1}, \omega_{2}\right)} .
$$

This finishes the proof of Theorem 2 .

\section{Boundedness on weighted Herz-type Hardy spaces}

Now we turn to consider the behaviour of local Calderón-Zygmund type operators on the weighted Herz-type Hardy spaces. We begin with recalling some definitions.

In what follows, for $s \in \boldsymbol{R}$, let $[s]$ denote the greatest integer $\leq s$ if $s \geq 0$ or 0 if $s<0$. Define

$$
\begin{aligned}
\mathscr{A}_{\alpha, q}^{q_{\omega_{1}}, q_{\omega_{2}}}\left(\boldsymbol{R}^{n}\right)=\left\{\phi \in \mathscr{S}\left(\boldsymbol{R}^{n}\right):\right. & \sup _{|\beta| \leq\left\{q_{\omega_{1}} \alpha+n\left(q_{\omega_{2}} / q-1\right)\right]+1} \\
& \left.(1+|x|)^{\left[q_{\omega_{1}} \alpha+n\left(q_{\omega_{2}} / q-1\right)\right]+n+1}\left|D^{\beta} \phi(x)\right| \leq 1\right\},
\end{aligned}
$$

where $\mathscr{S}\left(\boldsymbol{R}^{n}\right)$ is the space of the Schwartz functions, $\beta=\left(\beta_{1}, \ldots, \beta_{n}\right) \in(\boldsymbol{N} \cup\{0\})^{n}$ and $D^{\beta}=\left(\partial / \partial x_{1}\right)^{\beta_{1}} \cdots\left(\partial / \partial x_{n}\right)^{\beta_{n}}$. Moreover, we define

$$
\|\phi\|_{\mathscr{A}_{\alpha, q}^{q \omega_{1} \cdot q \omega_{2}}\left(\boldsymbol{R}^{n}\right)} \equiv \sup _{x \in \boldsymbol{R}^{n}|\beta| \leq\left\{q_{\omega_{1}} \alpha+n\left(q_{\omega_{2}} / q-1\right)\right]+1}(1+|x|)^{\left[q_{\omega_{1}} \alpha+n\left(q_{\omega_{2}} / q-1\right)\right]+n+1}\left|D^{\beta} \phi(x)\right| .
$$

Let $\mathscr{S}^{\prime}\left(\boldsymbol{R}^{n}\right)$ be the space of Schwartz distributions. For $f \in \mathscr{S}^{\prime}\left(\boldsymbol{R}^{n}\right)$, we define

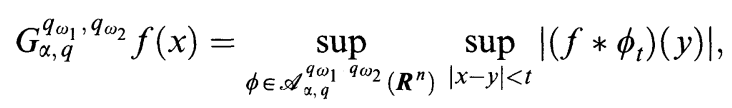

where $\phi_{t}(y)=t^{-n} \phi(y / t)$ for any $t>0 . \quad G_{\alpha, q}^{q_{\omega_{1}}, q_{\omega_{2}}} f$ is usually called to be the grand maximal function of $f$; see ([15], p. 90).

Now, we can give the definition of the weighted Herz-type Hardy space.

DEFINITION 3. Let $\alpha \in \boldsymbol{R}, \quad 0<p, \quad q \leq \infty, \quad \omega_{1} \in A_{q_{\omega_{1}}}\left(\boldsymbol{R}^{n}\right), \omega_{2} \in A_{q_{\omega_{2}}}\left(\boldsymbol{R}^{n}\right)$ and $1 \leq q_{\omega_{1}}, q_{\omega_{2}}<\infty$. The homogeneous weighted Herz-type Hardy space $H \dot{K}_{q}^{\alpha, p}\left(\omega_{1}, \omega_{2}\right)$ on $\boldsymbol{R}^{n}$ is defined by

$$
H \dot{K}_{q}^{\alpha, p}\left(\omega_{1}, \omega_{2}\right)=\left\{f \in \mathscr{S}^{\prime}\left(\boldsymbol{R}^{n}\right): G_{\alpha, q}^{q_{\omega_{1}}, q_{\omega_{2}}} f \in \dot{K}_{q}^{\alpha, p}\left(\omega_{1}, \omega_{2}\right)\right\},
$$


and

$$
\|f\|_{H K_{q}^{\alpha, p}\left(\omega_{1}, \omega_{2}\right)}=\left\|G_{\alpha, q}^{q_{\omega_{1}}, q_{\omega_{2}}} f\right\|_{K_{q}^{\alpha, p}\left(\omega_{1}, \omega_{2}\right)} .
$$

The non-homogeneous weighted Herz-type Hardy space $H K_{q}^{\alpha, p}\left(\omega_{1}, \omega_{2}\right)$ on $\boldsymbol{R}^{n}$ is defined by

$$
H K_{q}^{\alpha, p}\left(\omega_{1}, \omega_{2}\right)=\left\{f \in \mathscr{S}^{\prime}\left(\boldsymbol{R}^{n}\right): G_{\alpha, q}^{q_{\omega_{1}}, q_{\omega_{2}}} f \in K_{q}^{\alpha, p}\left(\omega_{1}, \omega_{2}\right)\right\},
$$

and

$$
\|f\|_{H K_{q}^{\alpha p}\left(\omega_{1}, \omega_{2}\right)}=\left\|G_{\alpha, q}^{q_{\omega_{1}}, q_{\omega_{2}}} f\right\|_{K_{q}^{\alpha, p}\left(\omega_{1}, \omega_{2}\right)} .
$$

If $\omega_{1} \equiv \omega_{2} \equiv 1$, we will denote $H \dot{K}_{q}^{\alpha, p}\left(\omega_{1}, \omega_{2}\right)$ and $H K_{q}^{\alpha, p}\left(\omega_{1}, \omega_{2}\right)$ simply by $H \dot{K}_{q}^{\alpha, p}\left(\boldsymbol{R}^{n}\right)$ and $H K_{q}^{\alpha, p}\left(\boldsymbol{R}^{n}\right)$ which are studied by [2], [4], [13] and so on when $\alpha$ and $p$ take some special values. If $\omega_{1}, \omega_{2} \in A_{1}\left(\boldsymbol{R}^{n}\right)$, the above Hardy spaces are studied by Lu and Yang in [12].

Applying Theorem 1, we can obtain the following relation between the weighted Herz space and the weighted Herz-type Hardy space. We omit the details.

THEOREM 3. Let $0<p \leq \infty, \quad 1<q<\infty, \quad \omega_{1} \in A_{q_{\omega_{1}}}\left(\boldsymbol{R}^{n}\right), \omega_{2} \in A_{q_{\omega_{2}}}\left(\boldsymbol{R}^{n}\right)$ and $1 \leq q_{\omega_{1}}<\infty$. Then $H \dot{K}_{q}^{\alpha, p}\left(\omega_{1}, \omega_{2}\right)=\dot{K}_{q}^{\alpha, p}\left(\omega_{1}, \omega_{2}\right)$ and $H K_{q}^{\alpha, p}\left(\omega_{1}, \omega_{2}\right)=$ $K_{q}^{\alpha, p}\left(\omega_{1}, \omega_{2}\right)$ provided either of the following holds:

(i) $\omega_{1}=\omega_{2}, 1 \leq q_{\omega_{1}} \leq q$ and $-n q_{\omega_{1}} / q<\alpha q_{\omega_{1}}<n\left(1-q_{\omega_{1}} / q\right)$;

(ii) $1 \leq q_{\omega_{2}} \leq q$ and $0<\alpha q_{\omega_{1}}<n\left(1-q_{\omega_{2}} / q\right)$.

Thus, the interesting case of the Herz-type Hardy space is $\alpha \geq\left(1-q_{\omega_{2}} / q\right) /$ $q_{\omega_{1}}$. For these spaces, we can establish their atomic decomposition.

Definition 4. Let $\omega_{1} \in A_{q_{\omega_{1}}}\left(\boldsymbol{R}^{n}\right), \omega_{2} \in A_{q_{\omega_{2}}}\left(\boldsymbol{R}^{n}\right), 1 \leq q_{\omega_{1}}, q_{\omega_{2}}<\infty, 1<q<$ $\infty, n\left(1-q_{\omega_{2}} / q\right) \leq \alpha q_{\omega_{1}}<\infty$ and the non-negative integer $s=\left[\alpha q_{\omega_{1}}+n\left(q_{\omega_{2}} / q-1\right)\right]$. A function $a(x)$ is said to be a central $\left(\alpha, q ; \omega_{1}, \omega_{2}\right)$-atom, if it satisfies

(i) $\operatorname{supp} a \subset B(0, r) \equiv\left\{x \in \boldsymbol{R}^{n}:|x| \leq r\right\}$ for some $r>0$,

(ii) $\|a\|_{L_{\omega_{2}}^{q}\left(\boldsymbol{R}^{n}\right)} \leq\left[\omega_{1}(B(0, r))\right]^{-\alpha / n}$,

(iii) $\int_{\boldsymbol{R}^{n}} a(x) x^{\beta} d x=0,|\beta| \leq s$.

When $\omega_{1}(x) \equiv \omega_{2}(x) \equiv 1$, we will denote the central $\left(\alpha, q ; \omega_{1}, \omega_{2}\right)$-atom simly by $(\alpha, q)$-atom.

Then by a similar proof to that of Theorem 1 in [12], we can show the following atomic decomposition; see also [4] and [13].

THEOREM 4. Let $0<p \leq \infty, 1<q<\infty, \omega_{1} \in A_{q_{\omega_{1}}}\left(\boldsymbol{R}^{n}\right), \omega_{2} \in A_{q_{\omega_{2}}}\left(\boldsymbol{R}^{n}\right), 1 \leq$ $q_{\omega_{1}}<\infty, 1 \leq q_{\omega_{2}} \leq q$ and $n\left(1-q_{\omega_{2}} / q\right) \leq \alpha q_{\omega_{1}}<\infty$. Then $f \in H \dot{K}_{q}^{\alpha, p}\left(\omega_{1}, \omega_{2}\right)$ (or $f \in H K_{q}^{\alpha, p}\left(\omega_{1}, \omega_{2}\right)$ ) if and only if $f=\sum_{k=-\infty}^{\infty} \lambda_{k} a_{k}$ (or $\left.f=\sum_{k=0}^{\infty} \lambda_{k} a_{k}\right)$ holds in $\mathscr{S}^{\prime}\left(\boldsymbol{R}^{n}\right)$, where $a_{k}$ is the central $\left(\alpha, q ; \omega_{1}, \omega_{2}\right)$-atom supported in $B_{k}$ and 
$\sum_{k=-\infty}^{\infty}\left|\lambda_{k}\right|^{p}<\infty$ (or $\left.\sum_{k=0}^{\infty}\left|\lambda_{k}\right|^{p}<\infty\right)$. Moreover,

$$
\begin{gathered}
\|f\|_{H K_{q}^{\alpha, p}\left(\omega_{1}, \omega_{2}\right)} \sim \inf \left\{\left(\sum_{k=-\infty}^{\infty}\left|\lambda_{k}\right|^{p}\right)^{1 / p}\right\} \\
\left(\text { or }\|f\|_{H K_{q}^{\alpha, p}\left(\omega_{1}, \omega_{2}\right)} \sim \inf \left\{\left(\sum_{k=0}^{\infty}\left|\lambda_{k}\right|^{p}\right)^{1 / p}\right\}\right),
\end{gathered}
$$

where the infimum is taken over all the above decompositions of $f$.

We remark that by the proof, Theorem 4 is still true if $\alpha>0$. Also, if $0<p \leq 1$, the central atom $a_{k}$ appearing in Theorem 1 does not necessarily support in $B_{k}$ and can support in any ball with the center at the origin.

Now, we have the following boundedness theorem on the local CalderónZygmund operator.

THEOREM 5. Let $T: \mathscr{S}\left(\boldsymbol{R}^{n}\right) \rightarrow \mathscr{S}^{\prime}\left(\boldsymbol{R}^{n}\right)$ be a linear and continuous operator. Suppose that the distribution kernel of $T$ coincides in the complement of the diagonal with a locally integrable function $k(x, y)$ satisfying

$$
|k(x, y)-k(x, 0)| \leq c \frac{|y|^{\delta}}{|x|^{n+\delta}}
$$

when $2|y|<|x|$ for some $\delta \in(0,1]$. Let $\omega_{1} \in A_{q_{\omega_{1}}}\left(\boldsymbol{R}^{n}\right), \omega_{2} \in A_{q_{\omega_{2}}}\left(\boldsymbol{R}^{n}\right), 1 \leq q_{\omega_{1}}<$ $\infty, \quad 1 \leq q_{\omega_{2}} \leq q<\infty$ and $n\left(1-q_{\omega_{2}} / q\right) \leq \alpha q_{\omega_{1}}<n\left(1-q_{\omega_{2}} / q\right)+\delta$. If $T$ is bounded on $L_{\omega_{2}}^{q}\left(\boldsymbol{R}^{n}\right)$, then $T$ is also bounded from $H \dot{K}_{q}^{\alpha, p}\left(\omega_{1}, \omega_{2}\right)$ into $\dot{K}_{q}^{\alpha, p}\left(\omega_{1}, \omega_{2}\right)$ for any $p \in(0, \infty]$.

Proof. Let $f \in H \dot{K}_{q}^{\alpha, p}\left(\omega_{1}, \omega_{2}\right)$. By Theorem 4, we have $f=\sum_{k=-\infty}^{\infty} \lambda_{k} a_{k}$, where $a_{k}$ is the central $\left(\alpha, q ; \omega_{1}, \omega_{2}\right)$-atom supported in $B_{k}$ and

Write

$$
\left\{\sum_{k=-\infty}^{\infty}\left|\lambda_{k}\right|^{p}\right\}^{1 / p} \leq c\|f\|_{H K_{q}^{\alpha, p}\left(\omega_{1}, \omega_{2}\right)} .
$$

$$
\begin{aligned}
\|T f\|_{K_{q}^{\alpha, p}\left(\omega_{1}, \omega_{2}\right)}= & \left\{\sum_{k=-\infty}^{\infty}\left[\omega_{1}\left(B_{k}\right)\right]^{\alpha p / n}\left\|\chi_{k} T f\right\|_{L_{\omega_{2}}^{q}\left(\boldsymbol{R}^{n}\right)}^{p}\right\}^{1 / p} \\
\leq & c\left\{\sum_{k=-\infty}^{\infty}\left[\omega_{1}\left(B_{k}\right)\right]^{\alpha p / n}\left(\sum_{l=-\infty}^{k-4}\left|\lambda_{l}\right|\left\|\chi_{k} T a_{l}\right\|_{L_{\omega_{2}}^{q}\left(\boldsymbol{R}^{n}\right)}\right)^{p}\right\}^{1 / p} \\
& +c\left\{\sum_{k=-\infty}^{\infty}\left[\omega_{1}\left(B_{k}\right)\right]^{\alpha p / n}\left(\sum_{l=k-3}^{\infty}\left|\lambda_{l}\right|\left\|\chi_{k} T a_{l}\right\|_{L_{\omega_{2}}^{q}\left(\boldsymbol{R}^{n}\right)}\right)^{p}\right\}^{1 / p} \\
\equiv & H_{1}+H_{2} .
\end{aligned}
$$


For $H_{2}$, by the $L_{\omega_{2}}^{q}\left(\boldsymbol{R}^{n}\right)$-boundedness of $T$ and $\alpha>0$, we have

$$
\begin{aligned}
H_{2} & \leq c\left\{\sum_{k=-\infty}^{\infty}\left[\omega_{1}\left(B_{k}\right)\right]^{\alpha p / n}\left(\sum_{l=k-3}^{\infty}\left|\lambda_{l}\right|\left\|a_{l}\right\|_{L_{\omega_{2}}^{q}\left(\boldsymbol{R}^{n}\right)}\right)^{p}\right\}^{1 / p} \\
& \leq c\left\{\sum_{k=-\infty}^{\infty}\left[\omega_{1}\left(B_{k}\right)\right]^{\alpha p / n}\left(\sum_{l=k-3}^{\infty}\left|\lambda_{l}\right|\left[\omega_{1}\left(B_{l}\right)\right]^{-\alpha / n}\right)^{p}\right\}^{1 / p} \\
& \leq c\left\{\sum_{k=-\infty}^{\infty}\left(\sum_{l=k-3}^{\infty}\left|\lambda_{l}\right| 2^{(k-l)} \delta_{\alpha}\right)^{p}\right\}^{1 / p} \\
& \leq c\left\{\sum_{l=\infty}^{\infty}\left|\lambda_{l}\right|^{p}\right\}^{1 / p} \\
& \leq c\|f\|_{H K_{q}^{\alpha p}\left(\omega_{1}, \omega_{2}\right)}
\end{aligned}
$$

where $\delta>0$ depends only on $n$ and $A_{q_{\omega_{1}}}\left(\boldsymbol{R}^{n}\right)$-constant of $\omega_{1}$, and we have omitted some similar computational techniques to those used in the estimates for $E_{1}$ and $E_{3}$.

To estimate $H_{1}$, for $x \in R_{k}$ and $l \leq k-4$, we have

$$
\begin{aligned}
\left|T a_{l}(x)\right| & \leq \int_{B_{l}}|k(x, y)-k(x, 0)|\left|a_{l}(y)\right| d y \\
& \leq c \int_{B_{l}} \frac{|y|^{\delta}}{|x|^{n+\delta}}\left|a_{l}(y)\right| d y \\
& \leq c 2^{-k(n+\delta)+l \delta} \int_{B_{l}}\left|a_{l}(y)\right| d y \\
& \leq c 2^{-k(n+\delta)+l \delta}\left\|a_{l}\right\|_{L_{\omega_{2}}^{q}\left(R^{n}\right)}\left(\int_{B_{l}}\left[\omega_{2}(x)\right]^{-1 /(q-1)} d x\right)^{1 / q^{\prime}} \\
& \leq c 2^{(l-k)(n+\delta)}\left[\omega_{1}\left(B_{l}\right)\right]^{-\alpha / n}\left[\omega_{2}\left(B_{l}\right)\right]^{-1 / q} .
\end{aligned}
$$

Thus, by $\alpha>0, \omega_{1} \in A_{q_{\omega_{1}}}\left(\boldsymbol{R}^{n}\right)$ and $\omega_{2} \in A_{q_{\omega_{2}}}\left(\boldsymbol{R}^{n}\right)$, we have

$$
\begin{aligned}
H_{1} & \leq c\left\{\sum_{k=-\infty}^{\infty}\left(\sum_{l=-\infty}^{k-4}\left|\lambda_{l}\right|\left[\frac{\omega_{1}\left(B_{k}\right)}{\omega_{1}\left(B_{l}\right)}\right]^{\alpha / n}\left[\frac{\omega_{2}\left(B_{k}\right)}{\omega_{2}\left(B_{l}\right)}\right]^{1 / q} 2^{(l-k)(n+\delta)}\right)^{p}\right\}^{1 / p} \\
& \leq c\left\{\sum_{k=-\infty}^{\infty}\left(\sum_{l=-\infty}^{k-4}\left|\lambda_{l}\right| 2^{(k-l)\left(q_{\omega_{1}} \alpha+n q_{\omega_{2}} / q-n-\delta\right)}\right)^{p}\right\}^{1 / p} \\
& \leq c\left\{\sum_{l=-\infty}^{\infty}\left|\lambda_{l}\right|^{p}\right\}^{1 / p} \\
& \leq c\|f\|_{H K_{q}^{\alpha, p}\left(\omega_{1}, \omega_{2}\right)}
\end{aligned}
$$


since $\alpha q_{\omega_{1}}+n q_{\omega_{2}} / q-n-\delta<0$, where we have omitted some similar computational techniques to the before.

This finishes the proof of Theorem 5.

If $q_{\omega_{1}}=q_{\omega_{2}}=1$ and $\alpha q_{\omega_{1}}=n\left(1-q_{\omega_{2}} / q\right)+\delta$, then we have the following weak boundedness theorem which can be regarded as the weighted version of Theorem 3 in [7].

THEOREM 6. Let $T$ and $k$ be the same as in Theorem 5. Let $0<p \leq 1$, $\omega_{1}, \omega_{2} \in A_{1}\left(\boldsymbol{R}^{n}\right), \quad 1<q<\infty$ and $\alpha=n(1-1 / q)+\delta$. If $T$ is bounded from $L_{\omega_{2}}^{q}\left(\boldsymbol{R}^{n}\right)$ into $W L_{\omega_{2}}^{q}\left(\boldsymbol{R}^{n}\right)$, then $T$ is also bounded from $H \dot{K}_{q}^{\alpha, p}\left(\omega_{1}, \omega_{2}\right)$ into $W \dot{K}_{q}^{\alpha, p}\left(\omega_{1}, \omega_{2}\right)$.

Proof. Let $f \in H \dot{K}_{q}^{\alpha, p}\left(\omega_{1}, \omega_{2}\right)$ and write $f$ as in the proof of Theorem 5 . We then have

$$
\begin{aligned}
\|T f\|_{W K_{q}^{\alpha, p}\left(\omega_{1}, \omega_{2}\right)}= & \sup _{\lambda>0} \lambda\left\{\sum_{k=-\infty}^{\infty}\left[\omega_{1}\left(B_{k}\right)\right]^{\alpha p / n}\left[D_{k, \omega_{2}}(\lambda, T f)\right]^{p / q}\right\}^{1 / p} \\
\leq & c \sup _{\lambda>0} \lambda\left\{\sum_{k=-\infty}^{\infty}\left[\omega_{1}\left(B_{k}\right)\right]^{\alpha p / n}\left[D_{k, \omega_{2}}\left(\lambda / 2, \sum_{l=-\infty}^{k-4} \lambda_{l} T a_{l}\right)\right]^{p / q}\right\}^{1 / p} \\
& +c \sup _{\lambda>0} \lambda\left\{\sum_{k=-\infty}^{\infty}\left[\omega_{1}\left(B_{k}\right)\right]^{\alpha p / n}\left[D_{k, \omega_{2}}\left(\lambda / 2, \sum_{l=k-3}^{\infty} \lambda_{l} T a_{l}\right)\right]^{p / q}\right\}^{1 / p} \\
\equiv & I_{1}+I_{2} .
\end{aligned}
$$

A desirable estimate for $I_{2}$ can be deduced from the boundedness of $T$ from $L_{\omega_{2}}^{q}\left(\boldsymbol{R}^{n}\right)$ into $W L_{\omega_{2}}^{q}\left(\boldsymbol{R}^{n}\right)$; we omit the details.

For $I_{1}$, by (3), for $x \in R_{k}$, we have

$$
\begin{aligned}
\left|\sum_{l=-\infty}^{k-4} \lambda_{l} T a_{l}(x)\right| & \leq c \sum_{l=-\infty}^{k-4}\left|\lambda_{l}\right| 2^{(l-k)(n+\delta)}\left[\omega_{1}\left(B_{l}\right)\right]^{-\alpha / n}\left[\omega_{2}\left(B_{l}\right)\right]^{-1 / q} \\
& \leq \frac{c}{\left[\omega_{1}\left(B_{k}\right)\right]^{\alpha / n}\left[\omega_{2}\left(B_{k}\right)\right]^{1 / q}} \sum_{l=-\infty}^{k-4}\left|\lambda_{l}\right| \\
& \leq \frac{c}{\left[\omega_{1}\left(B_{k}\right)\right]^{\alpha / n}\left[\omega_{2}\left(B_{k}\right)\right]^{1 / q}}\|f\|_{H K_{q}^{\alpha, p}\left(\omega_{1}, \omega_{2}\right)}
\end{aligned}
$$

since $p \in(0,1]$ and $\alpha=n(1-1 / q)+\delta$.

Now, by a similar computation to that for $F_{1}$, we can easily obtain a desirable estimate for $I_{1}$.

This finishes the proof of Theorem 6 . 
To investigate the boundedness of the operator $T$ in Theorems 5 and 6 on the space $H \dot{K}_{q}^{\alpha, p}\left(\omega_{1}, \omega_{2}\right)$, we need $T$ satisfies the following cancellation property; see [14].

Definition 5. Let $T$ be a linear operator. We say $T^{*} 1=0$ if $\int_{R^{n}} T a(x) d x$ $=0$ for all compactly supported bounded measurable functions a such that $\int_{\boldsymbol{R}^{n}} a(x) d x=0$.

The following theorem is a strong version of Theorem 5 and generalize Theorem 4 in [7] to both non-convolutional type and the weighted version.

THEOREM 7. Let $T: \mathscr{S}\left(\boldsymbol{R}^{n}\right) \rightarrow \mathscr{S}^{\prime}\left(\boldsymbol{R}^{n}\right)$ be a linear and continuous operator. Assume that the distributional kernel, $k(x, y)$, of $T$ satisfies (2) for some $\delta \in(0,1]$. Let $\omega_{1} \in A_{q_{\omega_{1}}}\left(\boldsymbol{R}^{n}\right), \omega_{2} \in A_{q_{\omega_{2}}}\left(\boldsymbol{R}^{n}\right), 1 \leq q_{\omega_{1}}<\infty, 1 \leq q_{\omega_{2}}<q$ and $n\left(1-q_{\omega_{2}} / q\right) \leq$ $\alpha q_{\omega_{1}}<n\left(1-q_{\omega_{2}} / q\right)+\delta$. If $T$ is bounded on $L_{\omega_{2}}^{q}\left(\boldsymbol{R}^{n}\right)$ and $T^{*} 1=0$, then $T$ is also bounded on $H \dot{K}_{q}^{\alpha, p}\left(\omega_{1}, \omega_{2}\right)$ for any $p \in(0, \infty]$.

Proof. Let $f \in H \dot{K}_{q}^{\alpha, p}\left(\omega_{1}, \omega_{2}\right)$ and write $f$ as in the proof of Theorem 5. We then have

$$
\begin{aligned}
& \|T f\|_{H K_{q}^{\alpha, p}\left(\omega_{1}, \omega_{2}\right)} \\
& =\left\|G_{\alpha, q}^{q_{\omega_{1}}, q_{\omega_{2}}}(T f)\right\|_{K_{q}^{\alpha . p}\left(\omega_{1}, \omega_{2}\right)} \\
& =\left\{\sum_{k=-\infty}^{\infty}\left[\omega_{1}\left(B_{k}\right)\right]^{\alpha p / n}\left\|\chi_{k} G_{\alpha, q}^{q_{\omega_{1}}, q_{\omega_{2}}}(T f)\right\|_{L_{\omega_{2}}^{q}\left(\boldsymbol{R}^{n}\right)}^{p}\right\}^{1 / p} \\
& \leq c\left\{\sum_{k=-\infty}^{\infty}\left[\omega_{1}\left(B_{k}\right)\right]^{\alpha p / n}\left(\sum_{l=-\infty}^{k-4}\left|\lambda_{1}\right|\left\|\chi_{k} G_{\alpha, q}^{q_{\omega_{1}}, q_{\omega_{2}}}\left(T a_{l}\right)\right\|_{L_{\omega_{2}}^{q}\left(\boldsymbol{R}^{n}\right)}\right)^{p}\right\}^{1 / p} \\
& +c\left\{\sum_{k=-\infty}^{\infty}\left[\omega_{1}\left(B_{k}\right)\right]^{\alpha p / n}\left(\sum_{l=k-3}^{\infty}\left|\lambda_{1}\right|\left\|\chi_{k} G_{\alpha, q}^{q_{\omega_{1}}, q_{\omega_{2}}}\left(T a_{l}\right)\right\|_{L_{\omega_{2}}^{q}\left(\boldsymbol{R}^{n}\right)}\right)^{p}\right\}^{1 / p} \\
& \equiv J_{1}+J_{2} \text {. }
\end{aligned}
$$

Applying $L_{\omega_{2}}^{q}\left(\boldsymbol{R}^{n}\right)$-boundedness of both $G_{\alpha, q}^{q_{\omega_{1}}, q_{\omega_{2}}}$ and $T$, we easily deduce a desirable estimate for $J_{2}$.

For $J_{1}$, we first estimate $G_{\alpha, q}^{q_{\omega_{1}}, q_{\omega_{2}}}\left(T a_{l}\right)(x)$ for $x \in R_{k}$ and $l \leq k-4$. In this case, choosing any $\phi \in \mathscr{A}_{\alpha, q}^{q_{\omega_{1}}, q_{\omega_{2}}}\left(\boldsymbol{R}^{n}\right)$ with $\|\phi\|_{\mathscr{A}_{\alpha, q}^{q \omega_{1}, q \omega_{2}}\left(\boldsymbol{R}^{n}\right)} \leq 1$, for $x \in R_{k},|x-y|<$ $t$ and $l \leq k-4$, by $T^{*} 1=0$, we have

$$
\begin{aligned}
\left|\left(T a * \phi_{t}\right)(y)\right| & =\left|\int_{\boldsymbol{R}^{n}} \operatorname{Ta}_{l}(z) \frac{1}{t^{n}} \phi\left(\frac{y-z}{t}\right) d z\right| \\
& =\left|\int_{\boldsymbol{R}^{n}} \operatorname{Ta}_{l}(z) \frac{1}{t^{n}}\left(\phi\left(\frac{y-z}{t}\right)-\phi\left(\frac{y}{t}\right)\right) d z\right|
\end{aligned}
$$


406

SHANZHEN LU, Kôzô YABUTA AND DACHUN YANG

$$
\begin{aligned}
\leq & \frac{1}{t^{n}} \int_{|z|<2^{l+1}}\left|T a_{l}(z)\right|\left|\phi\left(\frac{y-z}{t}\right)-\phi\left(\frac{y}{t}\right)\right| d z \\
& +\frac{1}{t^{n}} \int_{2^{l+1} \leq|z|<|x| / 2} \cdots+\frac{1}{t^{n}} \int_{|z| \geq|x| / 2} \cdots \\
\equiv & L_{1}+L_{2}+L_{3} .
\end{aligned}
$$

By the mean value theorem and Hölder's inequality, we have

$$
\begin{aligned}
L_{1} & \leq \frac{1}{t^{n+1}}\left\|T a_{l}\right\|_{L_{\omega_{2}}^{q}\left(\boldsymbol{R}^{n}\right)}\left(\int_{|z|<2^{l+1}}\left[\omega_{2}(z)\right]^{-q^{\prime} / q}\left|\nabla \phi\left(\frac{y-\theta z}{t}\right)\right|^{q^{\prime}}|z|^{q^{\prime}} d z\right)^{1 / q^{\prime}} \\
& \leq c 2^{l}\left\|a_{l}\right\|_{L_{\omega_{2}}^{q}\left(\boldsymbol{R}^{n}\right)}\left(\int_{|z|<2^{l+1}}\left[\omega_{2}(z)\right]^{-q^{\prime} / q}(t+|y-\theta z|)^{-(n+1) q^{\prime}} d z\right)^{1 / q^{\prime}} \\
& \leq \frac{c 2^{l}}{|x|^{n+1}\left[\omega_{1}\left(B_{l}\right)\right]^{\alpha / n}}\left(\int_{|z|<2^{l+1}}\left[\omega_{2}(z)\right]^{-q^{\prime} / q} d z\right)^{1 / q^{\prime}} \\
& \leq \frac{c 2^{l(n+1)}}{|x|^{n+1}\left[\omega_{1}\left(B_{l}\right)\right]^{\alpha / n}\left[\omega_{2}\left(B_{l}\right)\right]^{1 / q}},
\end{aligned}
$$

where $\nabla=\left(\partial / \partial x_{1}, \ldots, \partial / \partial x_{n}\right), c$ is independent of $\phi, x, t$ and $y$, and $\theta \in(0,1)$. Here we used the inequalities

$$
\left(1+\frac{|y-\theta z|}{t}\right)^{n+1}\left|\nabla \phi\left(\frac{y-\theta z}{t}\right)\right| \leq c\|\phi\|_{\mathscr{A}_{\alpha, q}^{q} \omega_{1}, q_{\omega_{2}}\left(\boldsymbol{R}^{n}\right)} \leq c
$$

and $t+|y-\theta z| \geq|x-y|+|y-\theta z| \geq|x-\theta z| \geq|x| / 2$. Using the same estimates, we have

$$
\begin{aligned}
L_{2}= & \frac{1}{t^{n+1}} \int_{2^{l+1} \leq|z|<|x| / 2}\left|\int_{|u| \leq 2^{l}} a(u)(k(z, u)-k(z, 0)) d u\right| \\
& \times\left|\nabla \phi\left(\frac{y-\theta z}{t}\right)\right||z| d z \\
\leq & \frac{c}{t^{n+1}} \int_{2^{l+1} \leq|z|<|x| / 2}\left(\int_{|u|<2^{l}}|a(u)| \frac{|u|^{\delta}}{|z|^{n+\delta}} d u\right) \\
& \times\left|\nabla \phi\left(\frac{y-\theta z}{t}\right)\right||z| d z \\
\leq & c 2^{l \delta}\|a\|_{L^{1}\left(\boldsymbol{R}^{n}\right)} \frac{1}{t^{n+1}} \int_{2^{l+1} \leq|z|<|x| / 2}\left|\nabla \phi\left(\frac{y-\theta z}{t}\right)\right| \frac{1}{|z|^{n+\delta-1}} d z
\end{aligned}
$$




$$
\begin{aligned}
& \leq \frac{c 2^{l \delta}}{|x|^{n+1}}\|a\|_{L_{\omega_{2}}^{q}\left(\boldsymbol{R}^{n}\right)}\left(\int_{B_{l}}\left[\omega_{2}(x)\right]^{-1 /(q-1)} d x\right)^{1 / q^{\prime}} \\
& \times \int_{2^{l+1} \leq|z|<|x| / 2} \frac{1}{|z|^{n+\delta-1}} d z \\
& \leq \begin{cases}\frac{c 2^{l(n+1)}}{|x|^{n+1}\left[\omega_{1}\left(B_{l}\right)\right]^{\alpha / n}\left[\omega_{2}\left(B_{l}\right)\right]^{1 / q}} \ln \left(\frac{|x|}{2^{l+2}}\right), & \text { if } \delta=1 \\
\frac{c 2^{l(n+\delta)}}{|x|^{n+\delta}\left[\omega_{1}\left(B_{l}\right)\right]^{\alpha / n}\left[\omega_{2}\left(B_{l}\right)\right]^{1 / q}}, & \text { if } \delta \in(0,1),\end{cases}
\end{aligned}
$$

by $\omega_{2} \in A_{q}\left(\boldsymbol{R}^{n}\right)$. For $L_{3}$, we have

$$
\begin{aligned}
L_{3} \leq & \frac{1}{t^{n}} \int_{|z| \geq|x| / 2}\left|\int_{|u| \leq 2^{l}} a(u)(k(z, u)-k(z, 0)) d u\right| \\
& \times\left(\left|\phi\left(\frac{y-z}{t}\right)\right|+\left|\phi\left(\frac{y}{t}\right)\right|\right) d z \\
\leq & \frac{c 2^{l(n+\delta)}}{t^{n}\left[\omega_{1}\left(B_{l}\right)\right]^{\alpha / n}\left[\omega_{2}\left(B_{l}\right)\right]^{1 / q}} \int_{|z| \geq|x| / 2} \frac{1}{|z|^{n+\delta}}\left(\left|\phi\left(\frac{y-z}{t}\right)\right|+\left|\phi\left(\frac{y}{t}\right)\right|\right) d z \\
\leq & \frac{c 2^{l(n+\delta)}}{|x|^{n+\delta}\left[\omega_{1}\left(B_{l}\right)\right]^{\alpha / n}\left[\omega_{2}\left(B_{l}\right)\right]^{1 / q}} .
\end{aligned}
$$

Thus, for $x \in R_{k}$ and $l \leq k-4$, we have

$$
G_{\alpha, q}^{q_{\omega_{1}}, q_{\omega_{2}}}\left(T a_{l}\right)(x) \leq c_{\varepsilon} \frac{2^{l(n+\varepsilon)}}{|x|^{n+\varepsilon}\left[\omega_{1}\left(B_{l}\right)\right]^{\alpha / n}\left[\omega_{2}\left(B_{l}\right)\right]^{1 / q}}
$$

for any $\varepsilon \in(0,1)$ where $\delta=1$ and $\varepsilon=\delta$ when $\delta \in(0,1)$, where $c_{\varepsilon}$ is independent of $x$ and $l$. From this, it follows that

$$
\begin{aligned}
J_{1} & \leq c_{\varepsilon}\left\{\sum_{k=-\infty}^{\infty}\left(\sum_{l=-\infty}^{k-4}\left|\lambda_{l}\right| 2^{(l-k)(n+\varepsilon)}\left[\frac{\omega_{1}\left(B_{k}\right)}{\omega_{1}\left(B_{l}\right)}\right]^{\alpha / n} \times\left[\frac{\omega_{2}\left(B_{k}\right)}{\omega_{2}\left(B_{l}\right)}\right]^{1 / q}\right)^{p}\right\}^{1 / p} \\
& \leq c_{\varepsilon}\left\{\sum_{k=-\infty}^{\infty}\left(\sum_{l=-\infty}^{k-4}\left|\lambda_{l}\right| 2^{(l-k)\left(n+\varepsilon-\alpha q_{\omega_{1}}-q_{\omega_{2}} n / q\right)}\right)^{p}\right\}^{1 / p} \\
& \leq c_{\varepsilon}\left\{\sum_{k=-\infty}^{\infty}\left|\lambda_{l}\right|^{p}\right\}^{1 / p} \\
& \leq c_{\varepsilon}\|f\|_{H K_{q}^{\alpha, p}\left(\omega_{1}, \omega_{2}\right)}
\end{aligned}
$$


where we choose $\varepsilon \in(0,1)$ such that $n+\varepsilon>\alpha q_{\omega_{1}}+q_{\omega_{2}} n / q$ when $\delta=1$ and $\varepsilon=\delta$ when $\delta \in(0,1)$.

This finishes the proof of Theorem 7.

To discuss the extreme case, $\alpha q_{\omega_{1}}=n\left(1-q_{\omega_{2}} / q\right)+\delta$, of Theorem 7, we introduce the weighted weak Herz-type Hardy space $W H \dot{K}_{q}^{\alpha, p}\left(\omega_{1}, \omega_{2}\right)$.

Definition 6. Let $\alpha \in \boldsymbol{R}, 0<p, q \leq \infty, \omega_{1} \in A_{q_{\omega_{1}}}\left(\boldsymbol{R}^{n}\right), \omega_{2} \in A_{q_{\omega_{2}}}\left(\boldsymbol{R}^{n}\right)$ and $1 \leq q_{\omega_{1}}, q_{\omega_{2}}<\infty$. The homogeneous weighted weak Herz-type Hardy space $W H \dot{K}_{q}^{\alpha, p}\left(\omega_{1}, \omega_{2}\right)$ on $\boldsymbol{R}^{n}$ is defined by

$$
W H \dot{K}_{q}^{\alpha, p}\left(\omega_{1}, \omega_{2}\right)=\left\{f \in \mathscr{S}^{\prime}\left(\boldsymbol{R}^{n}\right): G_{\alpha, q}^{q_{\omega_{1}}, q_{\omega_{2}}} f \in W \dot{K}_{q}^{\alpha, p}\left(\omega_{1}, \omega_{2}\right)\right\}
$$

and

$$
\|f\|_{W H K_{q}^{\alpha, p}\left(\omega_{1}, \omega_{2}\right)}=\left\|G_{\alpha, q}^{q_{\omega_{1}}, q_{\omega_{2}}} f\right\|_{W K_{q}^{\alpha, p}\left(\omega_{1}, \omega_{2}\right)} .
$$

The non-homogeneous weighted weak Herz-type Hardy space ${ }^{\prime} H K_{q}^{\alpha, p}\left(\omega_{1}, \omega_{2}\right)$ on $\boldsymbol{R}^{n}$ is defined by

$$
W H K_{q}^{\alpha, p}\left(\omega_{1}, \omega_{2}\right)=\left\{f \in \mathscr{S}^{\prime}\left(\boldsymbol{R}^{n}\right): G_{\alpha, q}^{q_{\omega_{1}}, q_{\omega_{2}}} f \in W K_{q}^{\alpha, p}\left(\omega_{1}, \omega_{2}\right)\right\}
$$

and

$$
\|f\|_{W H K_{q}^{\alpha, p}\left(\omega_{1}, \omega_{2}\right)}=\left\|G_{\alpha, q}^{q_{\omega_{1}}, q_{\omega_{2}}} f\right\|_{W K_{q}^{\alpha, p}\left(\omega_{1}, \omega_{2}\right)} .
$$

If $\omega_{1}(x) \equiv \omega_{2}(x) \equiv 1$, we will denote $W H \dot{K}_{q}^{\alpha, p}\left(\omega_{1}, \omega_{2}\right)$ and $\operatorname{WHK}_{q}^{\alpha, p}\left(\omega_{1}, \omega_{2}\right)$ simply by $W H \dot{K}_{q}^{\alpha, p}\left(\boldsymbol{R}^{n}\right)$ and $W H K_{q}^{\alpha, p}\left(\boldsymbol{R}^{n}\right)$ which are introduced by $\mathrm{Hu}, \mathrm{Lu}$ and Yang in [7]. Obviously, $W H \dot{K}_{p}^{0, p}=W H K_{p}^{0, p}=W H_{\omega_{2}}^{p}\left(\boldsymbol{R}^{n}\right)$ for any $p \in(0, \infty)$ which are studied by Quek and Yang in [14]; see also [3], [9], [1] and [16].

The following theorem is the end case of Theorem 7 and generalize Theorem 5 in [7] to both the non-convolutional case and the weighted case.

THEOREM 8. Let $T, k, \omega_{1}, \omega_{2}$ and $q$ be the same as in Theorem 7 with $\delta \in$ $(0,1)$ and $q_{\omega_{1}}=q_{\omega_{2}}=1$. If $\alpha=n(1-1 / q)+\delta$ and $p \in(0,1]$, then $T$ is bounded from $H \dot{K}_{q}^{\alpha, p}\left(\omega_{1}, \omega_{2}\right)$ into $W H \dot{K}_{q}^{\alpha, p}\left(\omega_{1}, \omega_{2}\right)$.

Proof. Let $f \in H \dot{K}_{q}^{\alpha, p}\left(\omega_{1}, \omega_{2}\right)$ and write $f$ as in the proof of Theorem 5 . We then have

$$
\begin{aligned}
& \|T f\|_{W H K_{q}^{\alpha, p}\left(\omega_{1}, \omega_{2}\right)} \\
& =\left\|G_{\alpha, q}^{q_{\omega_{1}}, q_{\omega_{2}}}(T f)\right\|_{W K_{q}^{\alpha, p}\left(\omega_{1}, \omega_{2}\right)} \\
& =\sup _{\lambda>0} \lambda\left\{\sum_{k=-\infty}^{\infty}\left[\omega_{1}\left(B_{k}\right)\right]^{\alpha p / n}\left[D_{k, \omega_{2}}\left(\lambda, G_{\alpha, q}^{q_{\omega_{1}}, q_{\omega_{2}}}(T f)\right)\right]^{p / q}\right\}^{1 / p}
\end{aligned}
$$




$$
\begin{aligned}
& \leq c \sup _{\lambda>0} \lambda\left\{\sum_{k=-\infty}^{\infty}\left[\omega_{1}\left(B_{k}\right)\right]^{\alpha p / n}\left[D_{k, \omega_{2}}\left(\lambda / 2, \sum_{l=-\infty}^{k-4}\left|\lambda_{l}\right| G_{\alpha, q}^{q_{\omega_{1}}, q_{\omega_{2}}}\left(T a_{l}\right)\right)\right]^{p / q}\right\}^{1 / p} \\
& +c \sup _{\lambda>0} \lambda\left\{\sum_{k=-\infty}^{\infty}\left[\omega_{1}\left(B_{k}\right)\right]^{\alpha p / n}\left[D_{k, \omega_{2}}\left(\lambda / 2, \sum_{l=k-3}^{\infty}\left|\lambda_{l}\right| G_{\alpha, q}^{q_{\omega_{1}}, q_{\omega_{2}}}\left(T a_{l}\right)\right)\right]^{p / q}\right\}^{1 / p} \\
& \equiv M_{1}+M_{2} .
\end{aligned}
$$

By the $L_{\omega_{2}}^{q}\left(\boldsymbol{R}^{n}\right)$-boundedness of $G_{\alpha, q}^{q_{\omega_{1}}, q_{\omega_{2}}}$ and $T$, we easily obtain a desirable estimate for $M_{2}$.

For $M_{1}$, when $x \in R_{k}$, by (4), we have

$$
\begin{aligned}
& \sum_{l=-\infty}^{k-4}\left|\lambda_{l}\right| G_{\alpha, q}^{q_{\omega_{1}}, q_{\omega_{2}}}\left(T a_{l}\right)(x) \\
& \quad \leq c_{\delta} \sum_{l=-\infty}^{k-4}\left|\lambda_{l}\right| 2^{(l-k)(n+\delta)} \frac{1}{\left[\omega_{1}\left(B_{l}\right)\right]^{\alpha / n}\left[\omega_{2}\left(B_{l}\right)\right]^{1 / q}} \\
& \quad \leq \frac{c_{\delta}}{\left[\omega_{1}\left(B_{l}\right)\right]^{\alpha / n}\left[\omega_{2}\left(B_{l}\right)\right]^{1 / q}} \sum_{l=-\infty}^{k-4}\left|\lambda_{l}\right| 2^{(l-k)\left(n+\delta-\alpha q_{\omega_{1}}-n q_{\omega_{2}} / q\right)} \\
& \quad \leq \frac{c_{\delta}}{\left[\omega_{1}\left(B_{l}\right)\right]^{\alpha / n}\left[\omega_{2}\left(B_{l}\right)\right]^{1 / q}}\left(\sum_{l=-\infty}^{k-4}\left|\lambda_{l}\right|^{p}\right)^{1 / p} \\
& \quad \leq \frac{c_{\delta}}{\left[\omega_{1}\left(B_{l}\right)\right]^{\alpha / n}\left[\omega_{2}\left(B_{l}\right)\right]^{1 / q}}\|f\|_{H K_{q}^{\alpha, p}\left(\omega_{1}, \omega_{2}\right)},
\end{aligned}
$$

since $p \leq 1$ and $\alpha=n(1-1 / q)+\delta$.

Now, similar to the above, we can obtain a desirable estimate for $M_{1}$.

This finishes the proof of Theorem 8 .

Finally, we remark that if we assume more regularity on the kernel, we could extend the range of $\alpha$ in Theorems 5 and 6; and moreover, if $T$ satisfies higher vanishing moment conditions, the range of $\alpha$ in Theorems 7 and 8 can also be extended. We omit the details.

\section{REFERENCES}

[1] J. Alvarez, $H^{p}$ and weak $H^{p}$ contınuity of Calderón-Zygmund type operators, Fourier Analysis (W O. Bray et al eds.), Lecture Notes in Pure and Appl. Math. 157, Dekker, 1994, 17-34.

[2] Y Z. Chen and K. S. LaU, Some new classes of Hardy spaces, J. Funct. Anal., 84 (1989), $255-278$. 
[ 3 ] R. Fefferman and F Soria, The space weak $H^{1}$, Studia Math., 85 (1987), 1-16.

[4] J. García-Cuerva and M.-J. L. Herrero, A theory of Hardy spaces associated to Herz spaces, Proc. London Math. Soc., 69 (1994), 605-628.

[5] J. García-Cuerva and J. L. Rubio de Francia, Weighted Norm Inequalities and Related Topıcs, Notas de Math. 116, North Holland, Amsterdam, 1985.

[6] G. Hu, S. Lu AND D. YANG, The weak Herz spaces, J. Beijing Normal Univ. (Natur. Sc1.), 33 (1997), 27-34.

[ 7 ] G. Hu, S. Lu AND D. YANG, The applications of weak Herz spaces, Adv. in Math. (China), 26 (1997), 417-428.

[ 8 ] E. HERnÁNDEZ AND D. YANG, Interpolation of Herz spaces and applications, Math. Nachr., 205 (1999), 69-87

[9] H. LiU, The weak $H^{p}$ spaces on homogeneous groups, Lecture Notes in Math. 1494, Springer-Verlag, Berlin, 1991, 113-118.

[10] X. LI AND D. YANG, Boundedness of some sublinear operators on Herz spaces, Illinors J. Math., 40 (1996), 484-501.

[11] S. Lu AND D. YANG, The decomposition of the weighted Herz spaces on $\boldsymbol{R}^{n}$ and its applications, Sc1. Chına Ser. A, 38 (1995), 147-158.

[12] S. Lu AND D. YANG, The werghted Herz-type Hardy spaces and its applications, Scl. China Ser. A, 38 (1995), 662-673.

[13] S. Lu ANd D. YAng, The local versions of $H^{p}\left(\boldsymbol{R}^{n}\right)$ spaces at the origin, Studia Math., 116 (1995), 103-131.

[14] T. QUEK AND D. YANG, Calderón-Zygmund type operators on welghted weak Hardy spaces over $\boldsymbol{R}^{n}$, to appear in Acta Math. Sinica New Ser.

[15] E. M. Stern, Harmonic Analysis: Real-variable Methods, Orthogonality and Oscillatory Integrals, Princeton Univ. Press, Princeton, 1993.

[16] Y ZHANG, On Oscillatory Integral Operators, Ph.D. Thesıs, Beijing Normal Unıv., Beijing, 1990 (in Chınese).

SHANZHEN LU AND Dachun YANG

DepartMent of MatheMatics

BEIING NORMAL UNIVERSITY

BEIJING 100875

P.R. CHINA

E-mails: lusz@bnu.edu.cn

dcyang@bnu.edu.cn

Kôzô YaBuTA

SCHOOL OF SCIENCE

KWANSEI GaKUIN UNIVERSITY

UEGAHARA 1-1-155, NISHINOMIYA 662-8501

JAPAN

E-mail: yabuta@kwanse1.ac.jp 